Vol 10, Issue 6, 2017

\title{
CHEMOTHERAPY OF LEISHMANIASIS - A REVIEW
}

\author{
BORAH UTTAM*, DASH BISWAJIT, DASH SUVAKANTA
}

Department of Pharmaceutical Science, Girijananda Chowdhury Institute of Pharmaceutical Science, Azara, Guwahati, India. Email: borahuttam@yahoo.in

Received: 03 April 2017, Revised and Accepted: 20 April 2017

\section{ABSTRACT}

Leishmaniasis is a dismissed vector-borne tropical contamination thought to be an ailment of poor people. It is a standout among the most ignored tropical sickness as far as medication disclosure and improvement. Moved in neediness stricken nations inside Southeast Asia, East Africa, and Latin America, it is likewise endemic in a few Mediterranean nations. The administration of the heterogeneous disorders controlled by parasites having a place with the genus Leishmania is, especially troublesome in created, non-endemic nations attributable to the newness of doctors with clinical side effects, demonstrative conceivable outcomes, and accessible treatment choices. Most antileishmanial medications are very lethal and introduce resistance issues or require hospitalization, being along these lines not sufficient to the field. As of late changes have been accomplished by blend treatment, decreasing the time, and cost of treatment. Regardless, new medications are still direly required. This overview highlights the chemotherapeutic operators against leishmaniasis, their science, method of activity and the component of resistance in the parasite. Future viewpoints in the territory of new hostile to leishmanial sedate targets are likewise specified.

Keywords: Visceral leishmaniasis, Cutaneous leishmaniasis, Chemotherapy, Antileishmanial drugs.

(C) 2017 The Authors. Published by Innovare Academic Sciences Pvt Ltd. This is an open access article under the CC BY license (http://creativecommons. org/licenses/by/4. 0/) DOI: http://dx.doi.org/10.22159/ajpcr.2017.v10i6.18863

\section{INTRODUCTION}

Chemotherapy is the only compelling approach to treat leishmaniasis [1]. Chemotherapy against leishmaniasis is constructed primarily on toxic pentavalent antimonial created during the main portion of the most recent century. Amid the most recent decade, elective medications have turned out to be accessible and enlisted for use in a few nations: be that as it may, most improvements in chemotherapy against leishmaniasis have originated from re-definition and rescreening of officially recognized medicaments instead of from reasonable outline of medications. This is on the grounds that both the science of the parasite and the immunological reaction of the host are not yet surely knew [2].

The primary medication medicines prescribed for both visceral leishmaniasis (VL) and cutaneous leishmaniasis (CL) were initially presented more than 50 years prior. In any case, the position is changing and some new treatments are getting to be noticeably accessible. Lamentably, it now appears to be evident that the past desire to build up a solitary medication or medication plan to be viable against all types of leishmaniasis was excessively hopeful. Leishmanial species very inherently in their medication affectability; additionally the instinctive and cutaneous locales of disease force contrasting pharmacokinetic necessities on the medications to be utilized. The objective for chemotherapy is the intracellular amastigote that survives and partitions in tissue macrophages whereby bringing about the sickness. The requests for new antileishmanial drugs have been bolstered as of late by the exhibit of gained imperviousness to the pentavalent antimonial medications, the principal line chemotherapy. The most extreme issue answered to date is in Bihar, India. The idea of medication resistance in leishmaniasis is not clear - affectability to drugs must be assessed deliberately and considered in connection to the distinctions in inherent medication affectability among species and circumstances where leishmaniasis is an anthroponotic infection instead of a zoonotic illness. Be that as it may, there is trouble when a standard medication regimen stops to powerful. Treatment viability is additionally traded off when there is immunosuppression, specifically because of HIV co-contamination. This can prompt fuel of malady or rising up out of dormant contamination - the exhausted insusceptible capacity implies that standard chemotherapy is as often as possible unsuccessful [3].
Sodium stibogluconate (SSG) (pentostam) and meglumine antimoniate (glucantime) are kept on being utilized as the first-line parenteral treatment for a long time for a wide range of leishmaniasis. The fundamental symptom related with antimonials is medication resistance. In 1975 and 1996, there are 1223 new clinical entities which have entered the market yet just 11 were utilized for the treatment of diseases. As per as regular sources is worried, there are many medications have been discovered, such as lapachol (hydroxy napthoquinone derivative) plumbagin, and biplumbagin (napthoquinoid subordinate). Dispyrin, a napthoquinoid subsidiary acquired from Diospyros montana Roxb., is utilized against Leishmania donovani. The primary component related with this is that it is DNA topoisomerase-1 [4].

\section{CLASSIFICATION OF ANTILEISHMANIASIS DRUGS [5]}

The brief classification of anti-leshmaniasis drugs is given in Table 1.

\section{First-line treatment}

Pentavalent antimonials

The antimonial was initially marketed at 1945 [3]. The principal operators with a great remedial file, the $P$. antimonials (SbV), were presented in the 1940s as yet the backbone of treatment for all types of leishmaniasis [1]. The primary restriction related with pentavalent antimonial is resistance; however, the prerequisite for up to 28 days of parenteral administration, the variable viability against VL and CL, and the rise of critical resistance is all components constraining the medications' helpfulness [3].

\section{CLASSIFICATION OF ANTIMONIAL}

SSG (Pentostam)

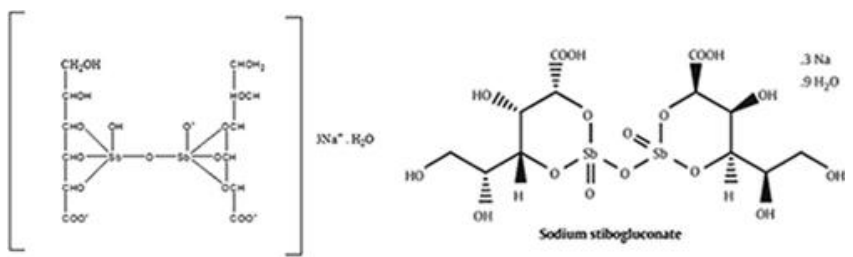


Table 1: Current drug used in the treatment of leishmaniasis

\begin{tabular}{ll}
\hline VL & \\
$\begin{array}{l}\text { First-line } \\
\text { drugs }\end{array}$ & SSG, meglumine antimoniate, Amp B, pentamidine \\
$\begin{array}{l}\text { Clinical } \\
\text { trails }\end{array}$ & $\begin{array}{l}\text { MT, paromomycin, sitamaquine, other Amp B } \\
\text { fL }\end{array}$ \\
$\begin{array}{l}\text { First-line } \\
\text { drugs }\end{array}$ & SSG, meglumine antimoniate, Amp B, pentamidine \\
$\begin{array}{l}\text { Clinical } \\
\text { trails }\end{array}$ & Paromomycin tropical formulation \\
& MT, paromomycin \\
& Imiquimod \\
& Antifungal azoles, i.e., ketoconazole, fluconazole, etc.
\end{tabular}

VL: Visceral leishmaniasis, CL: Cutaneous leishmaniasis, MT: Miltefosine,

SSG: Sodium stibogluconate, Amp B: Amphotericin B

\section{Meglumine antimonite (glucantime)}
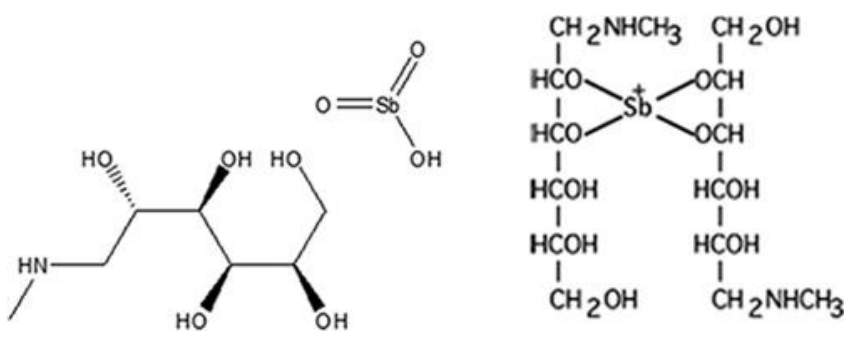

Antimonial is essentially used to be as first-line parenteral treatment for a wide range of leishmaniasis [6]. Essentially, they are utilized for the treatment of CL and instinctive leishmaniasis [7]. The primary reaction related with antimonial is medication resistance. There is an absence of viability [6].

These medications are related with extreme reactions, for example, cardiotoxicity, reversible renal inadequacy, pancreatitis, weakness, leukopenia, rashes, migraine, stomach torment, queasiness, spewing, thrombocytopenia, and transaminase height [8].

Poisonous pentavalent antimonial, which constitutes the backbone of treatment for leishmaniasis, has practically been deserted in India attributable to the absence of reaction of L. donovani against glucantime and N-methyl glucamine, in spite of the fact that they are as yet valuable in whatever is left of the world [2].

\section{Second-line treatment}

Polyene macrolide group of antibiotics

Amphotericin B (Amp B) (Fungizone)

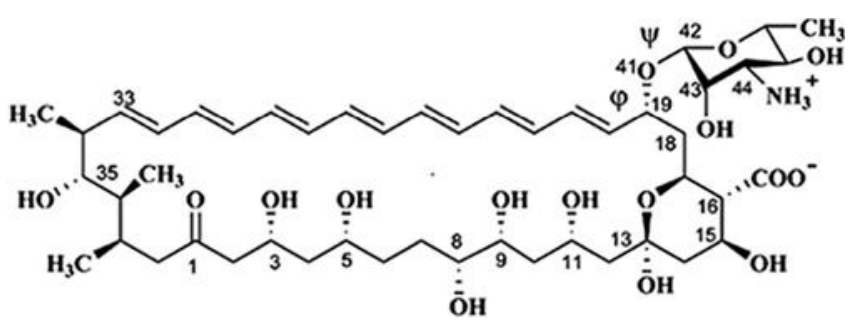

It is synthesized from actinomycetes microorganisms and compelling against pentavalent antimony-safe mucocutaneous ailment [9]. It follows up on sterols and phospholipids in cell films. It is compelling against parasites and leishmanial [10]. It has turned out to be exceptionally compelling for the treatment of antimonial-safe L. donovani VL [3]. Amp B is at present proposed as an option first-line treatment [11]. The principle restriction related with Amp B is harmfulness and its toxicity $[6,10]$.

\section{Lipid formulation of Amp B}

Middle Easterner plan is a colloidal scattering of lipid complex and is at first used to treat mycoses; however, nowadays, it is utilized for the treatment of instinctive leishmaniasis. It is for the most part follow-up on the reticuloendothelial arrangement of the body where leishmania reside. The fundamental favorable position of utilizing $A R a B$ over Amp B is poisonous quality. It primarily decreases the poisonous quality altogether. It is $95 \%$ viable. However, the principle impediment is the high cost. It is for the most part utilized for the treatment of VL-related AIDS and post dermal kala-azar $[1,12]$

Diamidine derivative: Pentamidine isethionate (pentam 300)<smiles>N=C(N)c1ccc(OCCCCCOc2ccc(C(=N)N)cc2)cc1</smiles>

Pentamidine

It is presented in 1952 [3]. It is considered as second-line treatment for instances of lethargy to antimony, in VL and CL [12]. Diverse creators have told distinctive system of activity of this medication. As per Papadopoulou et al., it might meddles with the vehicle and blend of polyamines which are required for the combination of trypanothione (N1, N8-bis-glutathionyl spermidine) [1]. Leandro have informed that it basically hinder DNA topoisomerase II [12]. Long haul regimen of highdosage pentamidine is presumably more dangerous than antimonial regimens and is in this manner utilized just when it is probably going to be more successful than antimony [1]. The fundamental impediment is the harmful impact on pancreas, kidney, or bone marrow. It might incite diabetes mellitus [7].

\section{Broad spectrum antibiotics: Aminoglycoside antibiotics}

Paromomycin sulfate: (Figure 1)

Paromomycin was initially isolated from filtrates of Streptomyces krestomuceticus in the 1950. At first, it was called as aminosidine. It is the main aminoglycoside with clinically essential antileishmanial movement. The antileishmanial properties of paromomycin were perceived by Kellina in 1961 and were affirmed by Neal et al. The fundamental instrument related with paromomycin is that it hinders protein amalgamation by communication with ribosomal RNA subunits [13]. Paromomycin in methylbenzethonium chloride ointment is utilized as a topical treatment for CL $[7,14]$.

Paromomycin has been utilized as a part of clinical trials for VL, CL. Paromomycin is in stage 2 clinical trials in India and Kenya

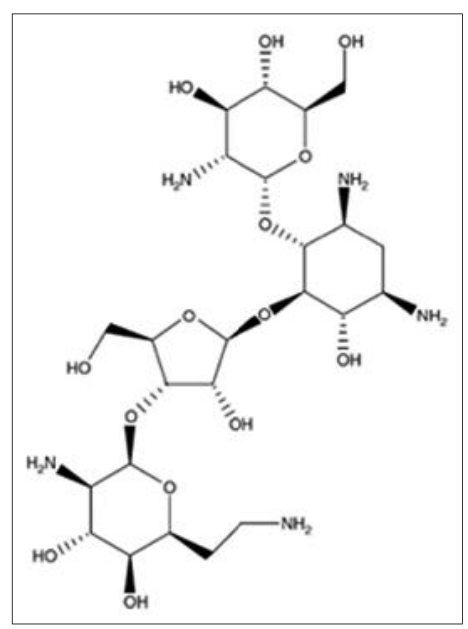

Fig. 1: Chemical structure of paromomycin 
which demonstrates that $90 \%$ of patients cured of VL. For CL it is utilized as a topical operator. As per El-On and partners in 1984 detailing containing 15\% PM and 12\% methylbenzethonium chloride (a skin-infiltrating operator) was compelling, against test CL prompted clinical trials. Paromomycin is utilized for neighborhood treatment [6].

Topical paromomycin is used against Leishmania major and Leishmania mexicana. It can be conjugated with antimonials to reduce the number of injections [7]. Paromomycin is currently in phase IV clinical trials against leishmaniasis [13]. The main side effect of paromomycin is inflammation and pigmentation [10].

\section{Nucleoside analogs}

\section{2,5 Azole derivatives}

The azoles (ketoconazole, fluconazole, and itraconazole) are dynamic option drugs, offering an appealing plausibility for the treatment of VL. Be that as it may, they should be better assessed [15].

- Ketoconazole

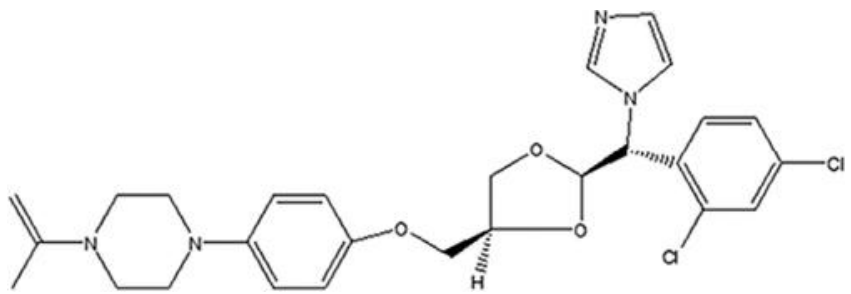

Ketoconazole acted by interferes with cell wall biosynthesis, inhibiting the 14 alpha-demethylation of lanosterol to ergosterol [13]. It has a variable cure rate for New World CL [6].

- Fluconazole

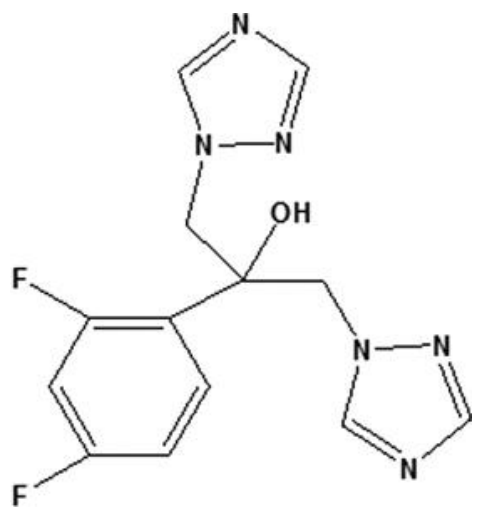

inhibiting the cytochrome P-450-mediated 14 alpha-demethylation of lanosterol, blocking ergosterol synthesis, and causing accumulation of 14 alpha-methyl sterols. Fluconazole is utilized orally [16]. It is active against $79 \%$ of patients infected with uncomplicated CL produced by L. major in Saudi Arabia [6]

\section{- Itraconazole}

It is a fresher synthetic triazole dioxolane derivative [10]. The azole mixes have unavoidably weaker activity than amphotericin since they act prior in the blend of ergosterol, by repressing the demethylation of lanosterol. They have not been reliably successful

with allopurinol or pentavalent antimonial. Fluconazole has, be that as it may, been effectively utilized for CL diseases brought on by L. major [12].

\section{Allylamine derivative}

- Terbinafine<smiles>C#CC=CCN(C)Cc1cccc2ccccc12</smiles>

Terbinafine is an engineered antifungal specialist of the allylamine class. Its antifungal action is because of impedance with ergosterol biosynthesis, particularly the profoundly specific hindrance of parasitic squalene epoxidase in contagious cell layers and the absence of resulting arrangement of lanosterol. Be that as it may, its utilization alone or with different medications in the treatment of cutaneous and instinctive VL, in vitro or in vivo (brought on by a few Leishmania animal categories), has created distinctive outcomes (Vannier-Santos et al., 1995; Rangel et al., 1996; Bahamdan et al., 1997; Urbina, 1997; Gangneux et al., 1999). As indicated by Melby et al. (2001), the hamster (Mesocricetus auratus) is a decent model for human VL. The medication had little or no leishmanicidal or leishmaniostatic impact on parasites display in the organs, for example, Leishmania chagasi and Leishmania infantum.

Rangel et al. (1996) have demonstrated the power of terbinafine agains Leishmania braziliensis. As indicated by Vannier-Santos et al. (1995), terbinafine alongside ketoconazole is utilized against L. amazonensis Urbina (1997) revealed the viability of terbinafine against $L$. braziliensis. The movement appeared by terbinafine is just in vitro [15].

\section{Phosphocholine analog: (Figure 2)}

Miltefosine (MT): (Impavido) (n-hexadecylphosphocholine) MT is an alky-lphosphocholine (ALP) imitative which was previous used as an anticancer drug [3,17] but nowadays castoff as antileishmanial drug, i.e., operative against both CL and VL (oral treatment.) $[3,18]$. It is used in opposition to antimony resistant parasites $[12,18]$. The antileishmanial movement of MT was at first found in the mid-1980s and the consequent exhibit of its viability in a few exploratory models in the mid-1990s to clinical trials and co-improvement of MT for leishmaniasis by an association between Asta Medica (now Zentaris) and WHO/TDR. After a Phase 3 trial, in which 282 out of 299 (94\%) VL patients were cured with an oral dosage of 2.5 mgkg 21 of MT

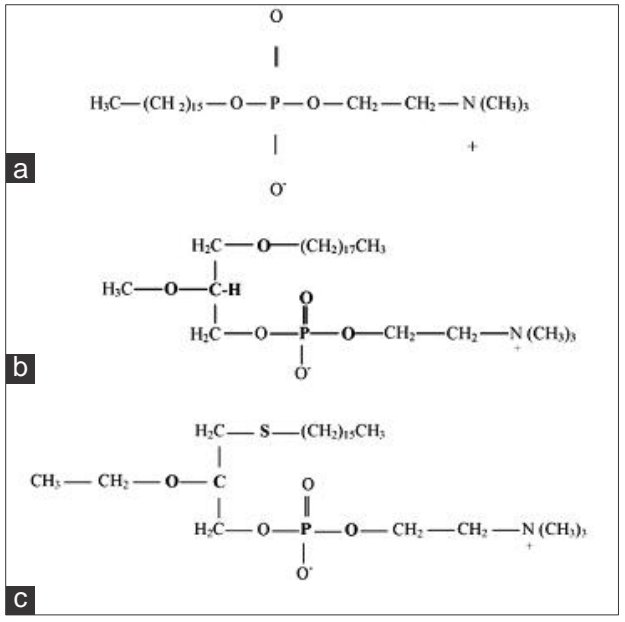

Fig. 2: Phospholipid drugs with antileishmanial activity. (a) Miltefosine, (b) edelfosine, (c) ilmofosine [19] 
day by day for 28 days. The real constraint of MT is teratogenicity and this bars its utilization in females of youngster bearing age [3].

It is utilized for the treatment of instinctive leishmaniasis. It is likewise utilized for the treatment of CL which is created by Leishmania vienna panamensis [6]. As of now, the most encouraging new pharmaceutical specialists are ALPs, for example, MT and edelfosine. MT is the principal oral medication ended up being exceedingly powerful against VL, including antimony-resistant cases. As of late, it has been liable to stage IV trials. There are, in any case, a few disadvantages, for example, potential teratogenicity and the possible improvement of medication resistance. These may in the long run, prompt the untimely end of its utilization. The method of activity against Leishmania is not yet settled [12].

\section{MT analog}

16-mercaptohexadecylphosphocholine

It is a thiol analog of MT. It is used for the oral treatment of human leishmaniasis. This simple exhibits the same leishmanicidal impact as the parent sedate against $L$. donovani promastigotes and Leishmania pifanoi axenic amastigotes. MT analogs with the lipophilic gatherings cyclohexylideneundecyl, adamantylideneundecyl, dodecylidene cyclohexyloxyethyl, or tetradecylidene-cyclohexyloxyethyl indicated more in vitro leishmanicidal movement than MT, and among them, just the two last mixes exhibited higher cytotoxicity in vivo than the parent tranquilize. Analogs with shorter chains, for example, phenoxyhexadecyl or 2-naphthyloxyethyl gatherings, were without activity. Regarding the polar head aggregate, it has been found that mixes with the terminal trimethylammonium assemble, as in phosphocholine, introduce higher in vitro action than the relating analogs with ammonium, N-methylpiperidinium, or N-methylmorpholinium terminal gatherings. Furthermore, couple phosphocholine subordinates have been synthesized which contain a particular columnist gather (test). That is the situation of MT analogs bearing the tetrafluorophenylazido photo labeling group20 or nitroxide paramagnetic gathering [18].

\section{5, 8-aminoquinolone}

Sitamaquine

The antileishmanial movement of this compound was initially distinguished in the 1970s at the Walter Reed Army Institute of Research. Sitamaquine is quickly used, shaping desethyl and 4-CH OH subsidiaries, which may be in charge of its action. Poisonous quality gives off an impression of being generally gentle, it causes mellow methemoglobinemia [3].

\section{Purine analog: Allopurinol}

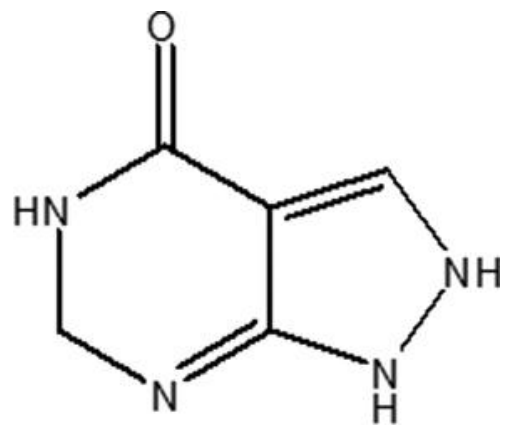

It was distinguished more than 30 years ago and on the grounds that it had oral bioavailability and was generally utilized for other clinical signs, it entered clinical trials for VL and CL. Be that as it may, the outcomes were frustrating. Allopurinol is utilized as a substrate by different proteins of the purine rescue pathway of trypanosomatids and is specifically fused into nucleotide intermediates and nucleic acids in the parasite. As of late, allopurinol has been considered as a feature of a support treatment for canine leishmaniasis against which it has suppressive movement [3]

\section{Sulfonamide derivative}

Dapsone<smiles>Nc1ccc(S(=O)(=O)c2ccc(N)cc2)cc1</smiles>

It is effective against Indian CL after 6 weeks of therapy. Sitamaquine is also utilized, as oral drug for the treatment of VL [6]. Dapsone perhaps act against leishmania by inhibition of choline incorporation into lecithin in the cell membrane, thus lessening phospholipid synthesis is first-line drug for the treatment of CL in India [10].

\section{Pyridinyl bisphosphonates}

- $\quad$ Risedronate (actonel)<smiles>O=P(O)(O)C(O)(Cc1cccnc1)P(=O)(O)O</smiles>

- Pamidronate

These are in extensive use in the treatment of bone disorders such as osteoporosis, have also shown action against leishmaniasis in experimental representations [3]

Mechanism action of anti-leishmanial drugs: (Table 2)

\section{Biaryls}

Arylanthranilodinitriles

Singh et al. have synthesized some arylanthranilodinitriles derivatives and exposed that a biaryl with trifluoromethyl group showed $83 \%$ hang-up against promastigotes and $70 \%$ inhibition against amastigotes of donovani

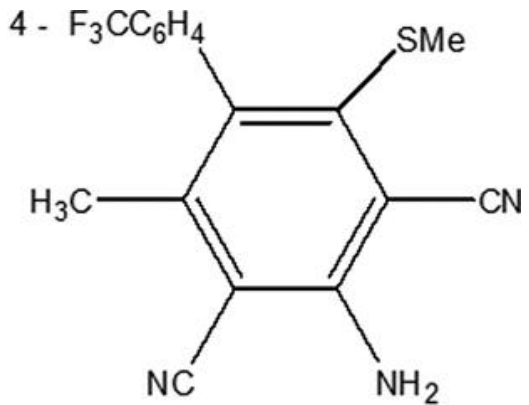


Table 2: MOA of antileishmanial drugs

\begin{tabular}{ll}
\hline Generic name of drug & Mechanism of action \\
\hline $\begin{array}{l}\text { Pentavalent antimonial } \\
\text { such as SSG, meglumine }\end{array}$ & $\begin{array}{l}\text { Structure of SSG is still not known despite its use for over } 50 \text { years. Activated within the amastigote, but not in the } \\
\text { promastigote, by converting to a lethal trivalent form. Activation mechanism is not known. Antileishmanial activity } \\
\text { might be due to action on host macrophage } \\
\text { Complexes with 24-substituted sterols. Such as ergosterol in cell membrane, thus causing pores which alter ion balance } \\
\text { and result in cell death }\end{array}$ \\
Pentamidine & $\begin{array}{l}\text { Accumulated by the parasite; effects include binding to kinetoplast DNA } \\
\text { Inhibit protein synthesis by binding to } 30 \text { seconds subunit of ribosomes, causing misleading and premature } \\
\text { Paromomycin }\end{array}$ \\
termination of mRNA translation \\
Possible inhibition of ether remodeling phosphatidylcholine biosynthesis \\
Sitamaquine
\end{tabular}

SSG: Sodium stibogluconate, MT: Miltefosine

$\mathrm{Ca}^{2+}$ Channel Blocker: (1,4-dihydropyridine derivative) Nimodipine<smiles>COCC(C)OC(=O)C1=C(C)N(C)C(C)C(C(=O)OC(C)C)=C1c1cccc([N+](=O)[O-])c1</smiles>

Nimodipine was highly effective against promastigotes and intracellular amastigotes of L. chagasi, with 50\% inhibitory concentration values of 81.2 and $21.5 \mu \mathrm{M}$, respectively. Nimodipine was about fourfold more effective than the standard pentavalent antimony against amastigotes and showed a selectivity index of 4.4 considering its mammalian cells toxicity. L. amazonensis and L. major promastigotes were also susceptible to nimodipine in a range concentration between 31 and $128 \mu \mathrm{M}$. Tempone et al. (2009) in their report showed that nimodipine affects mitochondrial functions, as no oxidation of MTT by dehydrogenases could be detected. Inhibition of oxygen consumption causes an increase in the intracellular reactive oxygen species, leading to a loss of mitochondrial membrane potential. This could have been a possible effect of nimodipine in Leishmania, resulting in enlarged mitochondria as observed in transmission electron microscopy. Further assays must be conducted to elucidate this mechanism and also to investigate the possibility of Leishmania apoptosis [21].

Verapamil: Recently, it was established that verapamil could efficiently reverse antimony resistance in L. donovani (Valiathan et al., 2006) [21].

\section{Macrolide antibiotics}

\section{Azithromycin}

Azithromycin is a macrolide antitoxin utilized for more than 40 years in numerous irresistible conditions, particularly those that influence the respiratory tract and in sexually transmitted sicknesses, which has potential movement against leishmaniasis. The macrolide acted by hindering protein union, separating the peptide from the ribosome

Azithromycin has indicated activity against L. major in vitro and in vivo, despite the fact that there has been no comprehension of its activity component. Utilizing it to treat patients, it demonstrated activity against leishmaniasis with remedial reaction slower than for antimonial operators 5 . In vitro analyses, azithromycin did not add to the phagocytosis of L. major, however it expanded the intracellular passing rate of amastigotes 8 . Another probability is that azithromycin has an immunomodulating impact, diminishing the quantity of amastigotes and enacting the neutrophils, keeping the generation of go-betweens and proinflammatory cytokines 5, 9. In vitro, it has additionally demonstrated viability against L. amazonensis, L. (V.) brazililensis, and L. chagasi in the focus $150 \mu \mathrm{g} / \mathrm{ml}$ [22].

\section{INTERFERON-GAMMA (IFN - 7)}

A few reviews have utilized IFN-gamma (IFN-7) as a subordinate to antimony treatment for instinctive leishmaniasis. Systemic IFN-7 is not viable by riser and has reactions, but rather it increases the adequacy of SbV treatment.

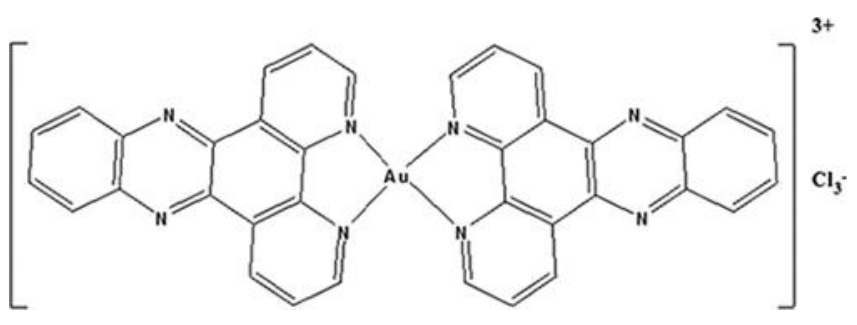

\section{TUFTSIN}

Tuftsin a tetrapeptide has been found to take an interest in a few organic capacities related with the invulnerable framework. It is created in the body from a particular cytophilic division of the protein (leukokinin) through a two-stage enzymatic preparing instrument. The tetrapeptide improves the phagocytic action of monocytes and macrophages. Particular restricting destinations for tuftsin have been uncovered to exist on macrophages. Tuftsin only ties to macrophages and potentiates their common executioner movement against pathogens. This makes tuftsin an alluring contender to be utilized as a ligand for focusing on medications to different macrophage-related maladies. Tuftsin-based focusing of SSG stacked liposomes was considered by L. donovani tainted hamsters for VL [23].

\section{PYRIMIDINES}

The library of substituted aryl pyrimidine subordinates was composed and incorporated at 2013. Every one of these mixes was screened in vitro by correspondent quality luciferase test for their antileishmanial property against intracellular amastigotes of $L$. donovani. Among them, 8 mixes indicated promising IC values $(0.5-12.9 \mu \mathrm{M})[24]$.

\section{CONCLUSION}

Examine went for recognizing and approving new medication targets is in one regard the most Leishmania particular piece of the medication disclosure prepare, albeit near reviews on Leishmania and trypanosomes have been powered by the yearning to locate a wide range antitrypanosomatid tranquilize. The cell science of Leishmania and mammalian cells gives the guarantee that a considerable lot of the parasite's proteins ought to be adequately not the same as anything in the host to be effectively misused as medication targets. 
Finding new subterranean insect leishmanial medications ought to be significantly less demanding than finding new anticancer medications. In this way, from the logical point of view there is much reason for positive thinking. To be sure, over numerous years, an entire exhibit of "intriguing medication targets" has been proposed - beyond any reasonable amount to list here. This claim has been made for some proteins that have been appeared to contrast from a mammalian partner in even a minor way. Shockingly, a few of these conceivable targets have been thoroughly approved. There are a few methodologies that can give such proof, the most capable being compound and hereditary approval.

Leishmania spp. has been creating wrecking dreariness and mortality since relic. Notwithstanding, in the $20^{\text {th }}$ century, the causative operator was found and inside 2 many years of its revelation, fruitful remedial specialists were found. The first being the trivalent and pentavalent antimoniates, which remained medication of leishmaniasis for the greater part a century. Shockingly, in late quarter of the twentieth century, lethargy to this medication began in Leishmania. The danger and expanding lethargy to antimoniates proceeded. Amid, these years a few laborers kept looking more secure mixes; however, little achievement was accomplished. Just as of late, Amp B, ordinarily utilized as an antifungal operator, was discovered best; however, even this medication has its own particular burdens, for example, nephrotoxicity. To decrease its nephrotoxicity, lipid details were readied; however, their cost wound up plainly excessively expensive to underdeveloped nations. No oral medication was accessible till 2004, when MT, an ALP compound, was discovered exceedingly powerful. In any case, it cannot be utilized as a part of pregnant women and kids. Furthermore, reports of resistance have begun developing. In this manner, we are left with no perfect viable medication, which is oral, safe, and financially savvy and does not create resistance. There are recommendations for mix treatment like on account of tuberculosis; however, cost of these medications should be cut down. However, the present circumstance for chemotherapy of leishmaniasis is more encouraging than any other time in recent memory, yet a leap forward is yet to come. It gives enough degree for discovering more current medications through seeing new metabolic pathways and medication targets.

\section{REFERENCES}

1. Papadopoulou B, Kündig C, Singh A, Ouellette M. Drug resistance in Leishmania: Similarities and differences to other organisms. Drug Resist Updat 1998;1(4):266-78.

2. Natera S, Machuca C, Padrón-Nieves M, Romero A, Díaz E, PonteSucre A. Leishmania spp.: Proficiency of drug-resistant parasites. Int J Antimicrob Agents 2007;29:637-42.

3. Croft SL, Coombs GH. Leishmaniasis - Current chemotherapy and recent advances in the search for novel drugs. Trends Parasitol 2003;19(11):502-8.

4. Hazra B, Golenser J, Nechemiya O, Bhattacharya S, Azzam T, Domb A, et al. Inhibitory activity of diospyrin derivatives against Leismania major parasites in vitro, Indian J Pharmacol 2002;34:422-7.

5. Croft SL, Seifert K, Yardley V. Current scenario of drug development for Leishmaniasis. Indian J Med Res 2006;123:399-410

6. Davis AJ, Kedzierski L. Recent advances in antileishmanial drug development. Curr Opin Investig Drugs 2005;6(2):163-9.

7. Markle WH, Makhoul K. Cutaneous leishmaniasis: Recognition and treatment. Am Fam Physician 2004;69(6):1455-60.

8. Visbal G, Marchán E, Maldonado A, Simoni Z, Navarro M. Synthesis and characterization of platinum-sterol hydrazone complexes with biological activity against Leishmania (L.) Mexicana. J Inorg Biochem 2008;102(3):547-54.

9. Baginski M, Czub J. Amphotericin B and its new derivatives - Mode of action. Curr Drug Metab 2009;10(5):459-69.

10. Dogra J. Current therapies for treatment of cutaneous leishmaniasis in India. Infection 1992;20(4):189-91.

11. Sereno D, Holzmuller P, Lemesre JL. Efficacy of second line drugs on antimonyl-resistant amastigotes of Leishmania infantum. Acta Trop 2000;74(1):25-31

12. Leandro C, Campino L. Leishmaniasis: Efflux pumps and chemoresistance. Int J Antimicrob Agents 2003;22(3):352-7.

13. Jhingran A, Chawla B, Saxena S, Barrett MP, Madhubala R. Paromomycin: Uptake and resistance in Leishmania donovani. Mol Biochem Parasitol 2009;164(2):111-7.

14. Davidson RN, den Boer M, Ritmeijer K. Paromomycin. Trans R Soc Trop Med Hyg 2009;103(7):653-60.

15. Simoes-Mattos MJ, Costa TD, Prata JR, Bevilaqua CM, Sidrim JJ, Rocha MF. Evaluation of terbinafine treatment in Leishmania chagasiinfected hamsters (Mesocricetus auratus). Vet Parasitol 2002;103:207-16.

16. Alrajhi AA, Ibrahim EA, De Vol EB, Khairat M, Faris RM, Maguire JH. Fluconazole for the treatment of cutaneous leishmaniasis caused by Leishmania major. N Engl J Med 2002;346(12):891-5.

17. Gupta L, Talwar A, Nishi, Palne S, Gupta S, Chauhan PM. Synthesis of marine alkaloid: 8,9-dihydrocoscinamide B and its analogues as Novel class of antileishmanial agents. Bioorg Med Chem Lett 2007; 17(14):4075-9.

18. Hornillos V, Saugar JM, de la Torre BG, Andreu D, Rivas L, Acuña AU, et al. Synthesis of 16-mercaptohexadecylphosphocholine, a miltefosine analog with leishmanicidal activity. Bioorg Med Chem Lett 2006;16(19):5190-3.

19. Croft SL, Engel J. Miltefosine - Discovery of the antileishmanial activity of phospholipid derivatives. Trans R Soc Trop Med Hyg 2006;100 Suppl 1:S4-8.

20. Singh FV, Vatsyayan R, Roy U, Goel A. Arylanthranilodinitriles: A new biaryl class of antileishmanial agents. Bioorg Med Chem Lett 2006;16(10):2734-7.

21. Tempone AG, Taniwaki NN, Reimão JQ. Antileishmanial activity and ultrastructural alterations of Leishmania (L.) chagasi treated with the calcium channel blocker nimodipine. Parasitol Res 2009;105(2):499-505.

22. Sampaio RN, Lucas IC, Filho AV. The use of azythromycin and $\mathrm{N}$-methyl glucamine for the treatment of cutaneous Leishmaniasis caused by Leishmania (Leishmania) amazonensis in C57BL6 mice. An Bras Dermatol 2009;84(2):125-8.

23. Chowdhary SJ, Chowdhary A, Kashaw S. Macrophage targeting: A strategy for leishmaniasis specific delivery. Int J Pharm Pharm Sci 2016;8(2):16-26

24. Sahu M, Siddiqui N. A review on biological importance of pyrimidines in the new era. Int J Pharm Pharm Sci 2016;8(5):8-14. 